\title{
ALTERNATIVA PARA CARACTERIZAČ̃̃O DA CONDUTIVIDADE HIDRÁULICA SATURADA DO SOLO UTILIZANDO PROBABILIDADE DE OCORRÊNCIA
}

\author{
Alternative of characterization to the soil hydraulic conductivity \\ utilizing probability of occurrence \\ Maria da Glória Bastos de Freitas Mesquita1, Sérgio Oliveira Moraes², \\ Fernanda Peruchi ${ }^{3}$, Maria de Carvalho Tereza ${ }^{3}$
}

\begin{abstract}
RESUMO
A Condutividade Hidráulica Saturada (Ksat) devido à sua importância em informar sobre a capacidade de transporte de água, solutos e substâncias químicas no solo deve ser bem caracterizada, pois de um modo geral, seu valor é utilizado nos cálculos de fluxos no solo. Com o objetivo de propor uma alternativa para caracterizá-la, a partir de uma série de dados, utilizou-se a função densidade de probabilidade lognormal para obter os valores da propriedade correspondentes aos níveis de 5 a $95 \%$ de probabilidade de ocorrência, visando descrever e indicar melhores valores a serem adotados como Ksat para a área considerada. Como resultado obtevese uma análise da variável em termos de probabilidade de ocorrência. Essa representação, na medida em que associa o nível de probabilidade ao valor adotado para a propriedade, permite ao pesquisador avaliar o risco na estimativa de medidas dependentes de Ksat, visto que esta propriedade no solo apresenta alta variabilidade.
\end{abstract}

Termos de indexação: Variabilidade, funções densidade de probabilidade, probabilidade de ocorrência, fluxo de água no solo.

\section{ABSTRACT}

The Saturated Hydraulic Conductivity of the soil (Ksat) due to its importance in inform about the capacity of transport of water, solutes and chemical substances in the soil should be well characterized, since in general, this value is used in calculations of flows in the soil. Aiming at proposing an alternative to characterize the Ksat, starting from a series of data, the function density of probability lognormal was used to obtain the values of the property which corresponde to the levels of occurrence probability from 5 to $95 \%$, in order to describe and to indicate better values to be adopted as Ksat for the considered area. As a result, it was obtained an analysis of the values of the variable in terms of occurrence probability. This representation, associating each value to a probability level, allows to the researcher to evaluate the error on estimation of measurements that depend on Ksat, due to the fact that, this property in the soil presents high variability.

Index terms: Variability, probability density functions, occurrence probability, water flux in soil.

(Recebido em 3 de janeiro de 2005 e aprovado em 25 de julho de 2006)

\section{INTRODUÇÃO}

Os processos de infiltração de água no solo, projetos de irrigação e drenagem, as perdas de solo por erosão e de substâncias químicas por lixiviação são geralmente relacionados ao fluxo de água nos solos, o qual influencia toda a utilização dos recursos solo e água.

Dentre as variáveis que influenciam este fluxo, a condutividade hidráulica do solo (K) se destaca. Ela é um parâmetro que representa a facilidade com que o solo transmite água. $\mathrm{O}$ valor máximo de condutividade hidráulica é quando o solo se encontra saturado, e é denominado condutividade hidráulica saturada (REICHARDT, 1990). A partir da condutividade hidráulica saturada (Ksat) e utilizando modelos matemáticos podese determinar a condutividade hidráulica do solo e assim obter informações sobre o movimento de água e solutos nos solos.

Há referências na literatura mostrando que os valores de condutividade hidráulica saturada em uma área específica podem ser muito distintos, assumindo grande amplitude total e elevados coeficientes de variação (BREJDA et al., 2000; COOKE et al., 1995; COOLEY, 1999; HANN \& ZHANG, 1996; KUTILEK \& NIELSEN, 1994; MESQUITA, 2001; MESQUITA et al., 2002; MOURA et al., 1999; PAZ et al., 1996; SMESRUD \& SELKER, 2001; WARRICK \& NIELSEN, 1980).

\footnotetext{
${ }^{1}$ Doutora, Professora - Departamento de Educação/DED - Universidade Federal de Lavras/UFLA - Cx. P. 3037 - $37200-000$ - Lavras, MG mgbastos@ufla.br

${ }^{2}$ Doutor, Professor - Departamento de Ciências Exatas - Escola Superior de Agricultura Luiz de Queiroz - ESALQ/USP - Cx. P. 9 - 13418-900 Piracicaba, SP - somoraes@esalq.usp.br

${ }^{3}$ Graduandas em Engenharia Florestal - Departamento de Educação/DED - Universidade Federal de Lavras/UFLA - Cx. P. 3037 - $37200-000$ - Lavras, MG.
} 
Isto pode ser um indicativo de que a propriedade não é representada por uma distribuição de probabilidade simétrica e, portanto nem sempre a média e/ou mediana podem ser assumidas como valores representativos de toda a área.

Embora haja o reconhecimento da grande variabilidade, observa-se que nos modelos matemáticos utilizados para descrição de processos que ocorrem nos solos, o valor de Ksat é considerado constante, sendo na maioria das vezes, utilizada a média aritmética e admitida a distribuição normal dos resultados sem questionar a validade e as implicações a que isto pode levar.

Como a Ksat é altamente variável espacialmente, a distribuição de probabilidade lognormal melhor se ajusta a seus dados observados, resultado este comprovado através de mais de um índice de comparação de ajuste por Logston et al. (1990), Mesquita (2001), Mesquita et al. (2002) e Warrick \& Nielsen (1980). Mas a dificuldade não se limita a identificar o tipo de distribuição empírica subjacente, há que se buscar a partir da distribuição pertinente, a melhor forma para caracterizar a variável.

Conforme relatado na literatura, alguma tentativa é feita no sentido de procurar critérios para o estabelecimento de valores realmente representativos da Ksat da área. Como alternativa, sugere-se a análise de probabilidade de ocorrência dos dados. Pode ser preferível caracterizar esta variável em termos de níveis de probabilidade de ocorrência, ao invés de utilizar um valor, como a média ou a mediana, para representar toda a área.

Portanto, o estudo da distribuição dos valores da propriedade e a escolha sobre a utilização de um único valor para representar a Ksat da área devem ser feitos com maior atenção considerando as características de variabilidade da propriedade e procurando minimizar os erros.

Conduziu-se este trabalho com o objetivo de apresentar uma análise sobre a caracterização da condutividade hidráulica saturada do solo, baseada não na adoção de uma medida de posição, mas no conhecimento do nível de probabilidade de ocorrência associado a cada valor, a partir da função densidade de probabilidade lognormal, já demonstrada como a mais adequada para representar os valores de Ksat.

\section{MATERIAL E MÉTODOS}

Foram estudados três solos, com diferentes classes texturais: um Latossolo Vermelho-Amarelo distrófico típico (LVAd), textura argilo arenosa; um Latossolo Vermelho distroférrico típico (LVdf), textura muito argilosa; e, um Neossolo Quartzarênico órtico típico (RQo), textura arenosa, provenientes da Região Centro-Oeste do Estado de São Paulo, a $22^{\circ} 41^{\prime}$ de latitude Sul, $47^{\circ} 39^{\prime}$ de longitude Oeste, e altitude de $550 \mathrm{~m}$, aproximadamente. Foram coletadas amostras indeformadas, na camada de 0 a $0,20 \mathrm{~m}$ de profundidade, utilizando-se amostrador tipo Uhland e cilindro metálico com diâmetro e altura médios de $0,072 \mathrm{~m}$, tendo sido coletadas 70 amostras para o solo LVAd, e 30 amostras para cada um dos solos LVdf e RQo.

A determinação da condutividade hidráulica saturada foi realizada pelo método do permeâmetro de carga constante (YOUNGS, 1991). As amostras indeformadas preparadas foram colocadas em bandeja, à qual foi adaptado um gotejador de Mariotte, que permitiu que as amostras fossem embebidas lentamente, de baixo para cima, com água destilada e deaerada. Este processo foi realizado para evitar o problema de formação de bolsas de ar e para facilitar a dissolução do ar contido dentro da amostra, conforme discutido por Faybishenko (1995) e Moraes (1991), mais detalhes podem ser vistos em Mesquita (2001).

O tratamento estatístico dos dados constitui-se de uma análise dos resultados em termos de probabilidade de ocorrência e de métodos adequados de determinação de parâmetros populacionais, considerando os resultados seguindo uma distribuição lognormal (PARKIN \& ROBINSON, 1992; PARKIN et al., 1988).

\section{RESULTADOS E DISCUSSÃO}

Na Tabela 1, apresenta-se o resultado da determinação da probabilidade de ocorrência para os valores de Ksat em determinados níveis de ocorrência préestabelecidos, considerando as funções densidade de probabilidade normal e lognormal para o solo LVAd e pode ser interpretada da seguinte forma: existe $5 \%(0,05)$ de probabilidade de que ocorra valor de Ksat menor ou igual a $0,0043 \times 10^{-2} \mathrm{~m} \mathrm{~s}^{-1}, 10 \%$ de probabilidade de que ocorra valor de Ksat menor ou igual a $0,0055 \times 10^{-2} \mathrm{~m} \mathrm{~s}^{-1}, 15 \%$ de probabilidade de que ocorra valor de Ksat menor ou igual a $0,0064 \times 10^{-2} \mathrm{~m} \mathrm{~s}^{-1}$, e assim por diante, quando a estimativa é feita a partir da função densidade de probabilidade lognormal. Da mesma forma, existe uma probabilidade de $95 \%$ de que ocorra valor menor ou igual a $0,0375 \times 10^{-2} \mathrm{~m} \mathrm{~s}^{-1}$.

Observa-se que admitindo a normalidade, ter-se-á uma probabilidade de 5\% de que exista valor de Ksat menor que $-0,0037 \times 10^{-2} \mathrm{~m} \mathrm{~s}^{-1}$, ou seja, a utilização da função normal levaria à estimativa de valores negativos de Ksat, o que é impossível na prática, pois o menor valor admissível para Ksat em um solo seria zero, o qual indicaria que o solo não permite o fluxo de água. Isto, porém não foi observado, pois em todas as amostras analisadas houve fluxo de água. Esta e outras considerações, também feitas por Mesquita 
et al. (2002), confirmam a utilização da distribuição de probabilidades lognormal para a descrição da propriedade condutividade hidráulica do solo saturado.

Para o solo LVAd, a probabilidade de ocorrência do valor médio (média $=0,0157 \times 10^{-2} \mathrm{~m} \mathrm{~s}^{-1}$ ) é de aproximadamente $65 \%$, e a de ocorrência do valor mediano, $\left(\right.$ mediana $=0,012724 \times 10^{-2} \mathrm{~m} \mathrm{~s}^{-1}$ ), é de $50 \%$, considerando a função densidade de probabilidade lognormal. A média considerando a distribuição normal tem uma probabilidade de ocorrência de aproximadamente $50 \%$, o que permite inferir que se esta distribuição for adotada a probabilidade de ocorrer um valor médio de Ksat será menor do que se a distribuição lognormal for adotada, logo a distribuição normal estaria subestimando a Ksat da área.

A alta variabilidade leva a uma incerteza maior nas estimativas e nos processos de inferências estatísticas. Em termos mais específicos, maior incerteza resulta em amplos intervalos de confiança para estimativa de parâmetros de posição (por exemplo, a média ou a mediana) e de dispersão (por exemplo, o desvio padrão ou variância), os quais resultam em baixo poder associado aos testes de hipóteses, o que poderia inclusive inviabilizar a utilização destes testes para comparações sobre efeitos de diferentes tratamentos (PARKIN et al., 1988). Isto sugere que uma melhor quantificação da variabilidade, ou o reconhecimento da existência desta variabilidade seja necessário para otimizar a análise estatística e o processo experimental.

A escolha pela medida que deve ser usada para representar a Ksat da área, e assim poder utilizar os modelos matemáticos propostos para o cálculo de fluxos nos solos e as operações de manejo, pode se tornar complicada quando a variabilidade da propriedade não é considerada.

Uma opção seria a utilização de tabelas como a Tabela 1, associada a análise gráfica, como a Figura 1, evidenciando a probabilidade de ocorrência e os valores da propriedade, que poderiam auxiliar a escolha por um valor para se adotar como representativo da variável, associando a este valor a probabilidade e possibilitando a estimativa de cálculo de erro advindo deste valor.

Pela Figura 1, mostra-se a curva de freqüência acumulada maior que (eixo horizontal superior) e menor que (eixo horizontal inferior) para os resultados de Ksat para os solos RQo e LVdf considerando a função densidade de probabilidade lognormal.

Tabela 1 - Valores observados e estimados em determinados níveis de probabilidade de ocorrência (menor que), para a variável condutividade hidráulica saturada (Ksat, $\left.x 10^{-2} \mathrm{~m} \mathrm{~s}^{-1}\right)$, considerando as funções densidade de probabilidade (F.D.P) normal e lognormal, para o LVAd.

\begin{tabular}{cccc}
\hline Nível de Probabilidade & Valores & \multicolumn{2}{c}{ Valores Estimados } \\
\cline { 2 - 3 } de ocorrência $(\%)$ & Observados & F.D.P. Normal & F.D.P. Lognormal \\
\hline 5 & 0,0035 & $-0,0037$ & 0,0043 \\
10 & 0,0057 & 0,0006 & 0,0055 \\
15 & 0,0087 & 0,0034 & 0,0064 \\
20 & 0,0096 & 0,0057 & 0,0073 \\
25 & 0,0102 & 0,0078 & 0,0082 \\
30 & 0,0109 & 0,0095 & 0,0090 \\
35 & 0,0117 & 0,0112 & 0,0099 \\
40 & 0,0123 & 0,0127 & 0,0108 \\
45 & 0,0124 & 0,0142 & 0,0117 \\
50 & 0,0133 & 0,0157 & 0,0127 \\
55 & 0,0142 & 0,0172 & 0,0138 \\
60 & 0,0160 & 0,0187 & 0,0151 \\
65 & 0,0169 & 0,0202 & 0,0164 \\
70 & 0,0176 & 0,0219 & 0,0180 \\
75 & 0,0186 & 0,0237 & 0,0198 \\
80 & 0,0214 & 0,0256 & 0,0221 \\
85 & 0,0232 & 0,0279 & 0,0252 \\
90 & 0,0394 & 0,0308 & 0,0295 \\
95 & & 0,0351 & 0,0375 \\
\hline
\end{tabular}



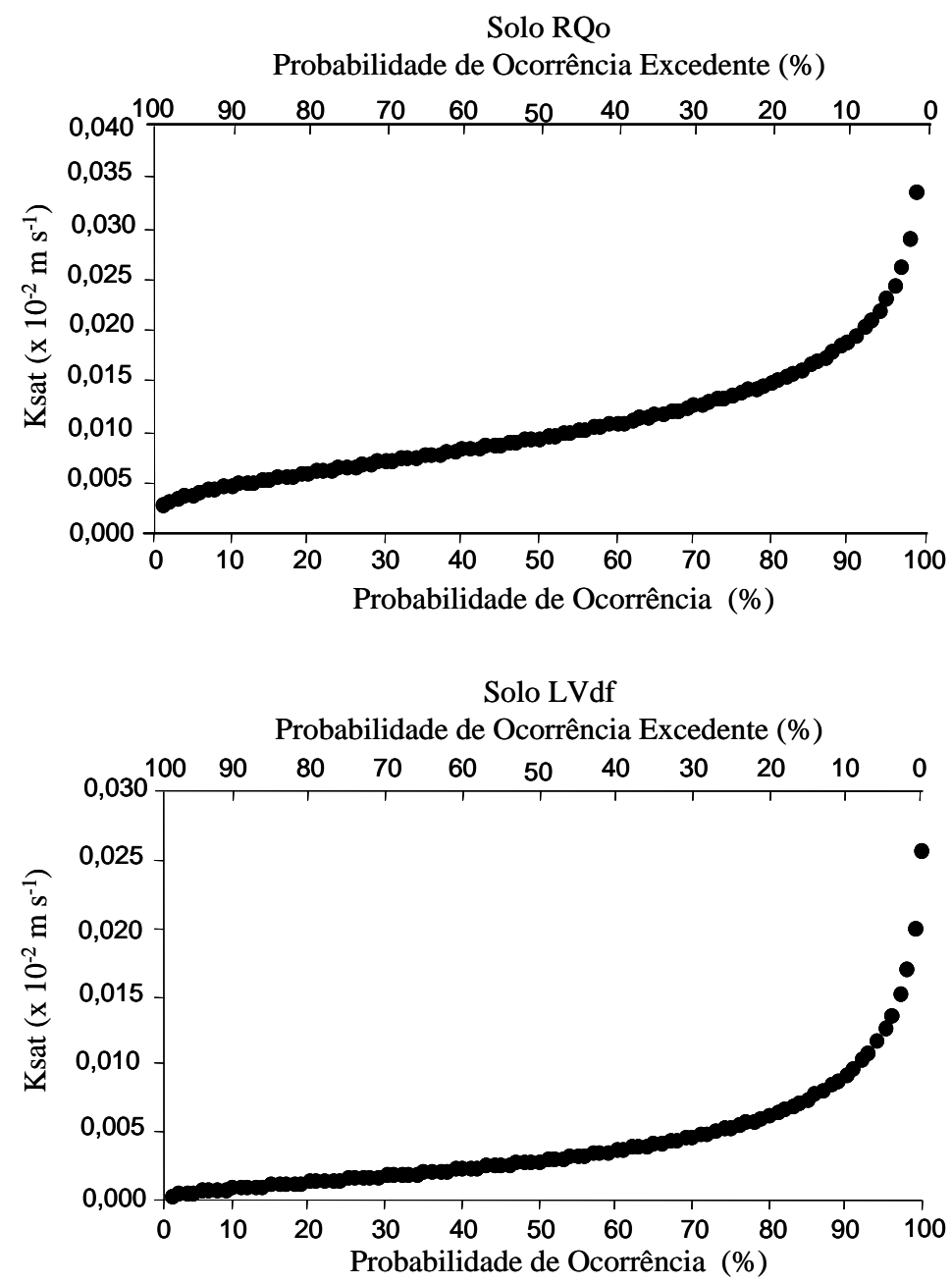

Figura 1 - Nível de probabilidade de ocorrência para os valores de Ksat para os solos RQo e LVdf, considerando a função densidade de probabilidade lognormal.

A partir da distribuição de probabilidades os resultados quantitativos podem ser assumidos com maior segurança, pois se torna possível estimar tanto a probabilidade como a variável correspondente, o que poderá fornecer informações mais claras e abrangentes.

Este tipo de alternativa é mais genérico, possibilitando ao pesquisador, liberdade de interpretação dos dados, assim como maior segurança na escolha do valor a ser adotado e na decisão sobre valores da propriedade que podem ser utilizados em estimativas de fluxos de água para grandes extensões de área. Assim, uma maior exploração dos métodos estatísticos e modelos matemáticos no tratamento dos resultados obtidos pelas análises de solos permitirá obter informações mais precisas, de acordo também com os autores Clausnitzer et al. (1998), Kutilek \& Nielsen (1994), Libardi et al. (1996), Mesquita et al. (2002), Moura et al. (1999) e Parkin et al. (1988).

\section{CONCLUSÃO}

A probabilidade de ocorrência associada à distribuição lognormal é uma alternativa para representação da condutividade hidráulica saturada, permitindo ao pesquisador avaliar o erro na estimativa de medidas de Ksat. 


\section{REFERÊNCIAS BIBLIOGRÁFICAS}

BREJDA, J. J.; MOORMAN, T. B.; SMITH, J. L.; KARLEN, D. L.;ALLAN, D. L.; DAO, T. H. Distribution and variability of surface soil properties at a regional scale. Soil Science Society of America Journal, Madison, v. 64, n. 3, p. 974$982,2000$.

COOKE, R. A.; MOSTAGHIMI, S.; WOESTE, F. Effect of hydraulic conductivity probability distribution function on simulated solute leaching. Water Environment Research, [S.1.], v. 67, n. 2, p. 159-168, 1995.

CLAUSNITZER, V.; HOPMANS, W.; STARR, J. L. Parameter uncertainty analysis of common infiltration models. Soil Science Society of America Journal, Madison, v. 62, p. 1477-1487, 1998.

COOLEY, R. L. Practical scheffe-type credibility intervals for variables of a groundwater model. Water Resources Research, [S.1.], v. 35, n. 1, p. 113-126, 1999.

FAYBISHENKO, B. A. Hydraulic behavior of quasisaturated soils in the presence of entrapped air: laboratory experiments. Water Resources Reserarch, [S.1.], v. 31, n. 10, p. 2421-2435, 1995.

HANN, C. T.; ZHANG, J. Impact of uncertain knowledge of model parameters on estimated runoff and phosphorus loads in the Lake Okeechobee basin. Transactions of the ASAE, [S.1.], v. 39, n. 2, p. 511-516, 1996.

KUTILEK, M.; NIELSEN, D. R. Soil hydrology. Berlin: Verlag, 1994. 370 p.

LIBARDI, P. L.; MANFRON, P. A.; MORAES, S. O.; TUON, R. L. Variabilidade da umidade gravimétrica de um solo hidromórfico. Revista Brasileira de Ciência do Solo, Campinas, v. 20, n. 3, p. 1-12, 1996.

LOGSTON, S. D.; ALLMARAS, R. R.; WU, L.; SWAN, J. B.; RANDALL, G. W. Macroporosity and its relation to saturated hydraulic conductivity under different tillage practices. Soil Science Society of America Journal, Madison, v. 54, p. 1096-1101, 1990.

MESQUITA, M. G. B. F. Caracterização estatística da condutividade hidráulica saturada do solo. 2001. 110 f. Tese (Doutorado) - Escola Superior de Agricultura Luiz de Queiroz, Universidade de São Paulo, Piracicaba, 2001.
MESQUITA, M. G. B. F.; MORAES, S. O.; CORRENTE, J. E. More adequate probability distributions to represent the saturated soil hydraulic conductivity. Scientia Agricola, Piracicaba, v. 59, n. 4, out./dez. 2002.

MORAES, S. O. Heterogeneidade hidráulica de uma terra roxa estruturada. 1991. $141 \mathrm{f}$. Tese (Doutorado) - Escola Superior de Agricultura Luiz de Queiroz, Universidade de São Paulo, Piracicaba, 1991.

MOURA, M. V. T.; LEOPOLDO, P. R.; MARQUES JÚNIOR, $\mathrm{S}$. Uma alternativa para caracterizar o valor da condutividade hidráulica em solo saturado. Irriga, Botucatu, v. 4, n. 2, p. 83-91, 1999.

PARKIN, T. B.; ROBINSON, J. A. Analysis of lognormal data. Advances in Soil Science, [S.1.], v. 20, p. 193-235, 1992.

PARKIN, T. B.; MEISINGER, J. J.; CHESTER, S. T.; STARR, J. L.; ROBINSON, J. A. Evaluation of statistical estimation methods for lognormal distributed variables. Soil Science Society of America Journal, Madison, v. 52, p. 323-329, 1988.

PAZ, A.; NEIRA, A.; CASTELAO, A. Soil water regime under pasture in the humid zone of Spain: validation of an empirical model and prediction of irrigation requirements. Agricultural Water Management, Ankeny, v. 29, n. 2, p. 147-161, 1996.

REICHARDT, K. A água em sistemas agrícolas. São Paulo: Manole, 1990. 188 p.

SMESRUD, J. K.; SELKER, J. S. Analytical solution for normal irrigation distribution parameters. Journal of Irrigation and Drainage Engineering, [S.1.], v. 127, n. 1, p. 45-48, 2001.

WARRICK, A. W.; NIELSEN, D. R. Spatial variability of soil physical properties in the field. In: HILLEL, D. (Ed.). Applications of soil physics. New York: Academic, 1980. p. 319-344.

YOUNGS, E. G. Hydraulic conductivity of saturated soils. In: SMITH, K. A.; MULLINS, C. E. (Eds.). Soil analysis: physical methods. New York: M. Dekker, 1991. p. 161-207. 